\title{
Spinal cord injury induces astroglial conversion towards neuronal lineage
}

\author{
Harun Najib Noristani ${ }^{1,2,3,4}$, Jean Charles Sabourin ${ }^{5}$, Hassan Boukhaddaoui ${ }^{4}$, Emilie Chan-Seng ${ }^{5,6}$, \\ Yannick Nicolas Gerber ${ }^{1,2,3,5}$ and Florence Evelyne Perrin ${ }^{1,2,3,4,5^{*}}$
}

\begin{abstract}
Background: Neurons have intrinsic capability to regenerate after lesion, though not spontaneously. Spinal cord injury (SCl) causes permanent neurological impairments partly due to formation of a glial scar that is composed of astrocytes and microglia. Astrocytes play both beneficial and detrimental roles on axonal re-growth, however, their precise role after $\mathrm{SCl}$ is currently under debate.

Methods: We analyzed molecular changes in astrocytes at multiple stages after two SCl severities using cell-specific transcriptomic analyses.

Results: We demonstrate that astrocyte response after injury depends on both time after injury and lesion severity. We then establish that injury induces an autologous astroglial transdifferentiation where over $10 \%$ of astrocytes express classical neuronal progenitor markers including Blll-tubulin and doublecortin with typical immature neuronal morphology. Lineage tracing confirmed that the origin of these astrocytes is resident mature, rather than newly formed astrocytes. Astrocyte-derived neuronal progenitors subsequently express GABAergic, but not glutamatergic-specific markers. Furthermore, we have identified the neural stem cell marker fibroblast growth factor receptor 4 (Fgfr4) as a potential autologous modulator of astrocytic transdifferentiation following SCl. Finally, we establish that astroglial transdifferentiation into neuronal progenitors starts as early as $72 \mathrm{~h}$ and continues to a lower degrees up to 6 weeks post-lesion.
\end{abstract}

Conclusion: We thus demonstrate for the first time autologous injury-induced astroglial conversion towards neuronal lineage that may represent a therapeutic strategy to replace neuronal loss and improve functional outcomes after central nervous system injury.

Keywords: Spinal cord injury, Astrocytes, Astrogliosis, Transdifferentiation, Cell specific transcriptomic

\section{Background}

Clinical symptoms linked to spinal cord injury (SCI) depend on the anatomical level and lesion severity, ranging from minor sensory/motor impairment to complete tetraplegia. Following SCI, a glial scar surrounds the lesion site constituting a real physicochemical barrier that is a major obstacle to axonal re-growth [1]. Glial scar is composed of two cell types, namely microglia and astrocytes. Although increased astrocyte reactivity (also referred as astrogliosis) is a prominent pathological hallmark after CNS lesion, its precise role after SCI has

\footnotetext{
* Correspondence: florence.perrin@inserm.fr

${ }^{1}$ University of Montpellier, Montpellier F-34095, France

${ }^{2}$ INSERM U1198, Place Eugène Bataillon CC105, 34095, Montpellier Cedex 5,

France

Full list of author information is available at the end of the article
}

been under debate for a long time [2]. Activated astrocytes over-express transcription factors that upsurges inflammation and inhibits axonal sprouting associated with worsening of functional recovery after SCI [3]. Concomitantly, astrocytes also play protective roles after injury via secretion of anti-inflammatory factors [4] and isolation of un-damaged tissue [5].

These studies highlight the beneficial and detrimental roles of astrocytes after SCI raising the question whether their contradictory responses may be due to differences in injury severity, time after injury or both. Detailed molecular analysis of pure astrocyte at acute and chronic stages after different lesion severities may accurately uncover their precise contribution in SCI pathophysiology. Indeed, recent studies, including our own, using cell- 
specific transcriptomic analyses have been critical to better understand the role of glial cells in numerous CNS pathophysiology [6-14].

Here, we used Aldh1l1-EGFP transgenic mice that express enhanced green fluorescent protein (eGFP) in astrocytes. Using fluorescence-activated cell sorting (FACS), we isolated pure astrocyte population and carried out RNA sequencing (RNA-Seq) to study the molecular signature of astrocytes after hemisection (HS) and full transection (FT) compared to that of non-injured (NI) controls. We have chosen FT since no regeneration can ever occur through the lesion site and lateral HS because limited spontaneous regeneration does occur due to the presence of undamaged tissue. We identified pronounced transcriptional deregulations in astrocytes following SCI that are driven by time post-injury and lesion severity. We also identified injury-induced astroglial transdifferentiation towards neuronal lineage where resident mature astrocytes overexpressed the neural stem cell marker fibroblast growth factor receptor 4 (Fgfr4), the neuronal progenitor markers $\beta$ III-tubulin and doublecortin (DCX). In addition, these astrocytes also transformed into classical neuronal progenitor-like morphology with bi-polar processes. Subsequently, astrocyte-derived neuronal progenitor cells expressed markers typically associated with mature GABAergic interneurons. More importantly, we demonstrate that astroglial transdifferentiation into neuronal progenitor starts as early as $72 \mathrm{~h}$ post-lesion and continues up to 6 weeks after both HS and FT. These data demonstrate a novel insight into glial cell plasticity after injury and provide therapeutic targets where autologous astroglial transdifferentiation could be enhanced to replace lost neurons and improve functional outcomes after SCI.

\section{Methods}

\section{Experimental procedures}

Transgenic mice expressing eGFP in astrocytes ((Aldh111EGFP)OFC789Gsat/Mmucd) were purchased from the Mutant Mouse Regional Resource Centre (MMRRC) and maintained on a Swiss Webster strain background. Aldehyde dehydrogenase 1 family member L1 is a specific panastrocytic marker [15]. Mice were housed in controlled conditions (hygrometry, temperature and $12 \mathrm{~h}$ light/dark cycle).

\section{Spinal cord injury}

Adult mice (12 weeks) were anesthetized by inhalation of isoflurane gas (1.5\%); laminectomy was performed and either a lateral hemisection (HS) or full transection (FT) was carried out under a microscope using a micro-scalpel (FST, Heidelberg, Germany), as described previously [16]. Lesions were done at thoracic 9 level to obtain hemi paraplegia (HS) or complete paraplegia (FT) while preserving full respiratory function.

\section{Postoperative cares}

Bladder was emptied manually twice daily until recovery of full sphincter control (for HS) or throughout the study period (for FT). Bodyweight was measured before surgery and daily throughout the 6 weeks period after injury.

\section{FACS}

Fluorescence Assisted Cell Sorting (FACS) was used to isolate pure populations of astrocytes from Aldh1l1EGFP transgenic mice. We used a $1 \mathrm{~cm}$-segment centered on the lesion site to isolate $\mathrm{eGFP}^{+}$astrocytes. For non-injured controls, the equivalent $1 \mathrm{~cm}$-segment of the thoracic spinal cord was used. Male Aldh1l1-EGFP mice were anesthetized with tribromoethanol $(500 \mathrm{mg} / \mathrm{kg})$ and intracardially perfused with RNAse-free $0.1 \mathrm{M}$ phosphate base saline (PBS, Invitrogen, Carlsbad, USA). Spinal cords were dissected and dissociated in $750 \mu \mathrm{PBS}, 100 \mu \mathrm{l}$ trypsin $13 \mathrm{mg} / \mathrm{ml}, 100 \mu \mathrm{l}$ hyaluronidase $7 \mathrm{mg} / \mathrm{ml}, 50 \mu \mathrm{l}$ kinurenic acid $4 \mathrm{mg} / \mathrm{ml}$ (all from Sigma Aldrich, Saint Louis, USA) and $20 \mu \mathrm{l}$ DNAseI $10 \mathrm{mg} / \mathrm{ml}$ (Roche, Rotkreuz, Switzerland) for $30 \mathrm{~min}$ at $37^{\circ} \mathrm{C}$. Gentle mechanic dissociation was carried out by pipetting. Cell suspension was sieved on a $40 \mu \mathrm{m}$ sieve (BD Biosciences, Franklin Lakes, USA), re-suspended in PBS-0.9 M sucrose and centrifuged for $20 \mathrm{~min}$ at $750 \mathrm{~g}$. The pellet was re-suspended in $500 \mu \mathrm{l}$ of 7 -aminoactinomycin D (7-AAD) $1 \mu \mathrm{l} / \mathrm{ml}$. Living astrocytes were sorted using FACS ARIA (BD Biosciences, Franklin Lakes, USA), equipped with a $488 \mathrm{~nm}$ Laser Sapphire 488-20. Size threshold was used to eliminate cellular debris. Isolated astrocytes were then centrifuged for $5 \mathrm{~min}$ at $700 \mathrm{~g}$ and re-suspended in $250 \mu \mathrm{l}$ of RLT lysis buffer (Qiagen, Maryland, USA) and $1 \%$ betamercaptoethanol. For flow cytometry analysis of eGFP and BIII-tubulin co-expression, after sieving and sucrose, cells were incubated for 20 min on ice in mouse anti $\beta I I I-$ tubulin in PBS (1:100; MAB1195; R\&D Systems, Minneapolis, USA). Cells were then centrifuged for $5 \mathrm{~min}$ at $400 \mathrm{~g}$, washed with cold PBS and incubated for $15 \mathrm{~min}$ on ice in Allophycocyanin (APC)-conjugated donkey anti mouse in PBS (1:100; 715-136-150; Jackson Immunoresearch, Carlsbad, USA). Controls were done using wild type mice (to show cell distribution without any staining), non-lesion segment alone (to show eGFP expression alone), wild type stained with $\beta$ III-tubulin (to show APC staining alone) as well as without primary antibody. Cells were centrifuged for $5 \mathrm{~min}$ at $400 \mathrm{~g}$, washed with cold $\mathrm{PBS}$ and re-suspended in 7-AAD before sorting.

\section{RNA sequencing}

Total RNA was isolated using RNeasy Mini Kit, (Qiagen, Maryland, USA) including DNAse treatment. The quality of starting RNA and amplified cRNA were tested (Agilent 2100 bioanalyzer, RNA 6000 Pico LabChip, Palo 
Alto, USA) and proceeded only if the RNA integrity number (RIN) was $>7$. Astrocytic RNA was isolated at 1 and 2 weeks after injury $(n=18$ for HS 1 week and $n=16$ for HS 2 weeks; $n=6$ for FT 1 week and $n=34$ for FT 2 weeks) as well as NI controls $(n=13)$. RNA-Seq was performed on the polyadenylated fraction of RNA. Three biological replicates were used for each of the mentioned time-points including NI control in all experimental conditions. $10 \mathrm{ng}$ of total RNAs were used for each sequencing library. Sequencing quality control was done using FastQC. The reads containing adapter sequences were removed with FASTX-Toolkit. The filtered reads were mapped with TopHat v2.0.9 software in order to use the UCSC mm10 release reference on new junctions and known annotations. Biological quality control and summarization were done using RSeQC v2.3.3, PicardTools v1.92 and SamTools v.1.18. The count data were prepared with HTSeq v.0.5.4p3. Data normalization and differential expression analysis was performed using $\mathrm{R} /$ Bioconductor edgeR software v.3.3.6. Very lowly expressed genes were filtered out whilst genes that achieved 10 counts in at least one condition were kept. The filtered data were normalized by the library size and differentially expressed genes were estimated using negative binomial general model statistics. To identify transcripts that were differentially expressed, we defined a criterion of a 2-fold and greater difference plus a significant $(p<0.05)$ false discovery rate (FDR). Statistics: $t$-test with un-equal variance. The $\mathrm{R} /$ Bioconductor MFuzz package v.2.18.0 was used for fuzzy clustering of the differentially expressed genes over time in both $\mathrm{HS}$ and FT compared to NI controls. Pathway analysis was done using MetaCore, as previously described [10].

\section{Primary antibodies for immunological staining}

Primary antibodies used included rabbit anti GFAP (1:1000; Z 0334; Dako, Glostrup, Denmark), mouse anti GFAP (1:1000; G3893; Sigma Aldrich, Saint Louis, USA), mouse anti MAP2 (1:500; M-1406; Sigma Aldrich, Saint Louis, USA), mouse anti-MBP (1:500; MAB 384; Millipore, California, USA), rabbit anti Iba1 (1:1000; 019-19741; Wako Pure Chemical Industries, Osaka, Japan), chicken anti GFP (1:1000; Ab13970; Abcam, Cambridge, UK), mouse anti ßIII-tubulin (1:500; MAB1195; R\&D Systems, Minneapolis, USA), goat anti Fgfr4 (1:500; AF2265; R\&D, Minneapolis, USA), rabbit anti GAD 65/67 (1:500; AB1511; Millipore, California, USA), guinea pig anti Tlx3/Rnx (1:500; kindly provided by Thomas Müller and Carmen Birchmeier, MaxDelbrück-Center, Berlin, Germany), mouse anti NeuN (1:500; MAB377; Millipore, California, USA), rabbit anti doublecortin (1:500; ab18723; Abcam; Cambridge, UK), rat anti BrdU (1:500; Ab6326; Abcam; Cambridge, UK) and mouse anti parvalbumine (1:500; P3088, Sigma Aldrich, Saint Louis, USA).

\section{Immunohistochemistry}

Hemisected and non-injured control female mice were anesthetized with tribromoethanol (500 mg/kg, i.p) and perfused intracardially with $0.1 \mathrm{M}$ PBS followed by $4 \%$ paraformaldehyde (PFA, Sigma Aldrich, Saint Louis, USA). Spinal cords were removed and post-fixed for $2 \mathrm{~h}$ in $4 \%$ PFA, cryoprotected in sucrose $30 \%$, included in Tissue Tek (Sakura, Alphen aan den Rijn, The Netherlands), frozen and kept at $-20{ }^{\circ} \mathrm{C}$ until processing. Frozen spinal cords were cut either longitudinally $(20 \mu \mathrm{m})$ or transversally $(14 \mu \mathrm{m})$. All sections were collected on super frost plus slides.

For fluorescence immunohistochemistry, slides were washed in PBS, treated for $20 \mathrm{~min}$ in $0.1 \mathrm{M}$ PBS containing lysine (20 mM, pH 7.2). Sections were then blocked for $1 \mathrm{~h}$ with $0.1 \mathrm{M}$ PBS containing bovine serum albumin (BSA, $1 \%$, Sigma Aldrich, Saint Louis, USA) and triton X-100 (0.1 \%, Fisher Scientific, Illkirch, France) followed by incubation in primary antibody for $48 \mathrm{~h}$ at $4{ }^{\circ} \mathrm{C}$. Slides were then washed in $0.1 \mathrm{M}$ PBS and were placed in corresponding secondary antibodies conjugated to Alexa 488, 594 or 633 (Vector Laboratories, Burlingame, USA and Millipore Bioscience Research Reagents, Massachusetts, USA). The nuclear stain, 4' ,6-diamidino-2-phenylindole dihydro-chloride (DAPI, $2 \mathrm{ng} / \mathrm{ml}$, Invitrogen, Massachusetts, USA) was used as a general nucleus marker. Sections were cover slipped using fluorescent mounting medium (DAKO, Denmark). For BrdU detection, sections were pre-incubated in $2 \mathrm{~N} \mathrm{HCl}$ for $30 \mathrm{~min}$ of DNA denaturation before fluorescence immunohistochemistry.

Morphometric fluorescent photographs were obtained using laser scanning inverted (Leica SP5, Mannheim, Germany) and (Zeiss 5 Live Duo, Oberkochen, Germany) confocal microscopy to unveil the co-expression of two proteins. $225 \times 225 \mu^{2}$ images were taken adjacent to the lesion center and the colocalization coefficient were measured between the two channels. 4-7 animals were used at each given time-point. Colocalization analysis was performed using the Carl Zeiss LSM-710-NLO software.

\section{Area fraction}

To determine Fgfr4 protein expression, the area fraction was measured in at least $1000 \mu^{2}$ surface area rostral and $1000 \mu \mathrm{m}^{2}$ caudal to the lesion sites at multiple timepoints after SCI. Area fraction is a sensitive and reliable method to measure expression level of a given signal and to detect changes caused by experimental manipulations. To avoid potential variation in staining intensity between different slides or animals, we had carried out immunostaining of all animals in parallel. Immunofluorescent- 
labelled spinal cord sections were imaged using constant exposure time in randomly selected longitudinal section with clearly visible lesion site (Zeiss, Oberkochen, Germany). Area fraction quantification was performed using ImageJ software (NIH, USA). Threshold was adjusted according to non-injured control and was kept constant for all images. Area fraction was analyzed individually both rostral and caudal to the lesion site. For non-injured controls, we analyzed Fgfr4 expression in equivalent surface area within the thoracic spinal cord segment. Five time-points after injury were analyzed for Fgfr4 expression level namely, 72 h, 1, 2, 4 and 6 weeks after lesion with 5 animals per groups.

\section{BrdU injection after SCI}

BrdU (Sigma Aldrich, Saint Louis, USA), was administered by intraperitoneal injections at $100 \mathrm{mg} / \mathrm{kg}$ dissolved in saline. Each animal was given a single daily injection immediately after lesion through 7 days after SCI.

\section{Statistical analysis}

One-way analysis of variance was used to compare differences over-time. Un-paired t-tests were used to compare differences between two independent groups. Significance was accepted at $\mathrm{p} \leq 0.05$. The data were analyzed using GraphPad Prism 4.0 (GraphPad Software, Inc, CA, USA). All data are shown as the mean \pm standard error of the mean (SEM).

\section{Results}

Time and severity-dependent astrocytic response after $\mathrm{SCl}$

Dual immunohistochemistry in Aldh1l1-EGFP mice using classical astroglial marker glial fibrillary acidic protein confirmed specific eGFP expression in astrocytes (Additional file 1: Figure S1a-c). No eGFP expression was observed in microglia/macrophages, oligodendrocytes and neurons (Additional file 1: Figure S1d-g). Using FACS we isolated pure astroglial populations with high RNA quality (Additional file 1: Figure S1h-k). We then carried out RNA-Seq analysis to uncover molecular changes in astrocytes at multiple stages after two SCI severities. Analysis of deregulated transcripts showed specific up-regulation of classical astroglia-specific marker genes (gfap and serpina3n) and no dysregulation in neuronal (nefl, Syt1, Gabra1, and Snap25) and oligodendrocytes (Mog, Sox10, Cx47 and Mbp) transcripts [14] that confirms sample purity (Additional file 2: Table S1).

We identified 61 and 124 DE genes in HS as well as 297 and 364 DE genes in FT groups at 1 week and 2 weeks, respectively (Fig. 1a, b). Comparisons of commonly deregulated genes over time revealed 39 and 58 DE genes in HS and FT groups, respectively (Fig. 1a-c). Comparisons between the two injury severities showed higher number of DE genes after FT compared to HS injury at all time-points (Fig. $1 \mathrm{a}-\mathrm{g}$ ). Pathway analysis identified $C 4 a$ and $C 4 b$ as the only transcripts associated with immune responses that were upregulated in HS group at 1 and 2 weeks post-injury (Fig. 1h). Simultaneously, at 2 weeks after HS, other genes linked to immune response and Il13 signaling via JAK-STAT pathway were down-regulated including Chitinase 3 L3 (Chi3l3), Mmp8 and Rsnb (Additional file 3: Table S2). On the other hand, at 1 week after FT astrocytes displayed pronounced up-regulation of numerous transcripts associated with immune response, inflammation and natural killer cell cytotoxicity (Fig. 1h, i). At 2 weeks after FT, astrocytes displayed down-regulation of transcripts involved in proteolysis and connective tissue degradation, mitotic cell division and cytoskeleton remodeling (Fig. 1j, k).

Taken together, these results suggest that (1) the number of DE genes in astrocytes are higher after FT compared to HS injury, (2) astrocytic response after injury depends on both time- and lesion severity, (3) at 1 week after HS astrocytes only marginally participate in inflammatory process followed by inhibition of immune response at 2 weeks after HS and (4) at 1 week after FT astrocytes promote immune response and inflammation, followed by inhibition of connective tissue degradation and reduced proliferation.

\section{Resident mature astrocytes express neuronal progenitors and GABAergic markers associated with neural stem cell marker Fgfr4 over-expression after SCI}

Pathway analysis in astrocytes identified "neural stem cell lineage" at 2 weeks after FT (Additional file 3: Table S2) through the up-regulation of the neuronal progenitor gene BIII-tubulin (Tubb3, also known as TUJ1, Additional file 4: Figure S2). Increased Tubb3 expression in astrocytes was observed at 1 and 2 weeks after HS and FT (Fig. 2b). Immunohistochemistry analysis confirmed BIII-tubulin expression in a sub-population of astrocytes located adjacent to micro-cavities within $750 \mu \mathrm{m}$ distance to the lesion site (Fig. $2 \mathrm{k}-\mathrm{r}$ ). $\beta$ III-tubulin expression in astrocytes was also associated with a change in cell morphology from typical stellate shape to classical neuronal progenitor cells with bipolar or multipolar processes (Fig. 2o-r). Astrocytes in non-injured controls showed no $\beta$ III-tubulin expression (Fig. $2 c-j$ ). We then used FACS to deepen eGFP/ $/$ III-tubulin co-expression analysis at single-cell level (Fig. $2 \mathrm{~s}-\mathrm{w}$ ). Unlike non-injured controls, eGFP/ $\beta$ III-tubulin co-expressing cells were specifically observed after HS (Fig. 2v \& w). FACS analysis revealed that at 2 weeks after HS injury $14.96 \%$ of astrocytes expressed $\beta$ III-tubulin (Fig. $2 \mathrm{w}$ ). To examine the origin of eGFP/ $\beta$ III-tubulin co-expressing cells, we then carried out BrdU injection in Aldh1l1-EGFP mice 


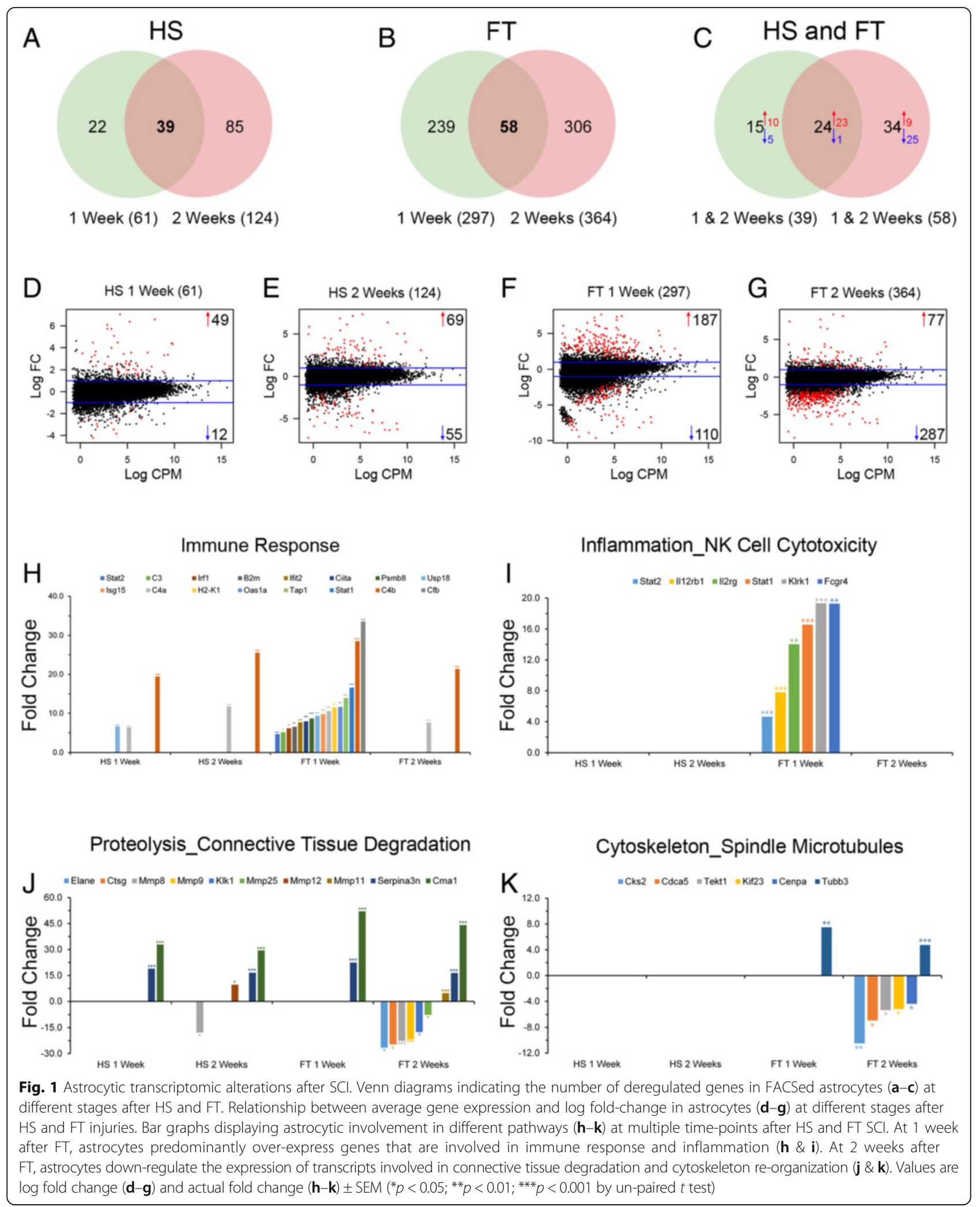




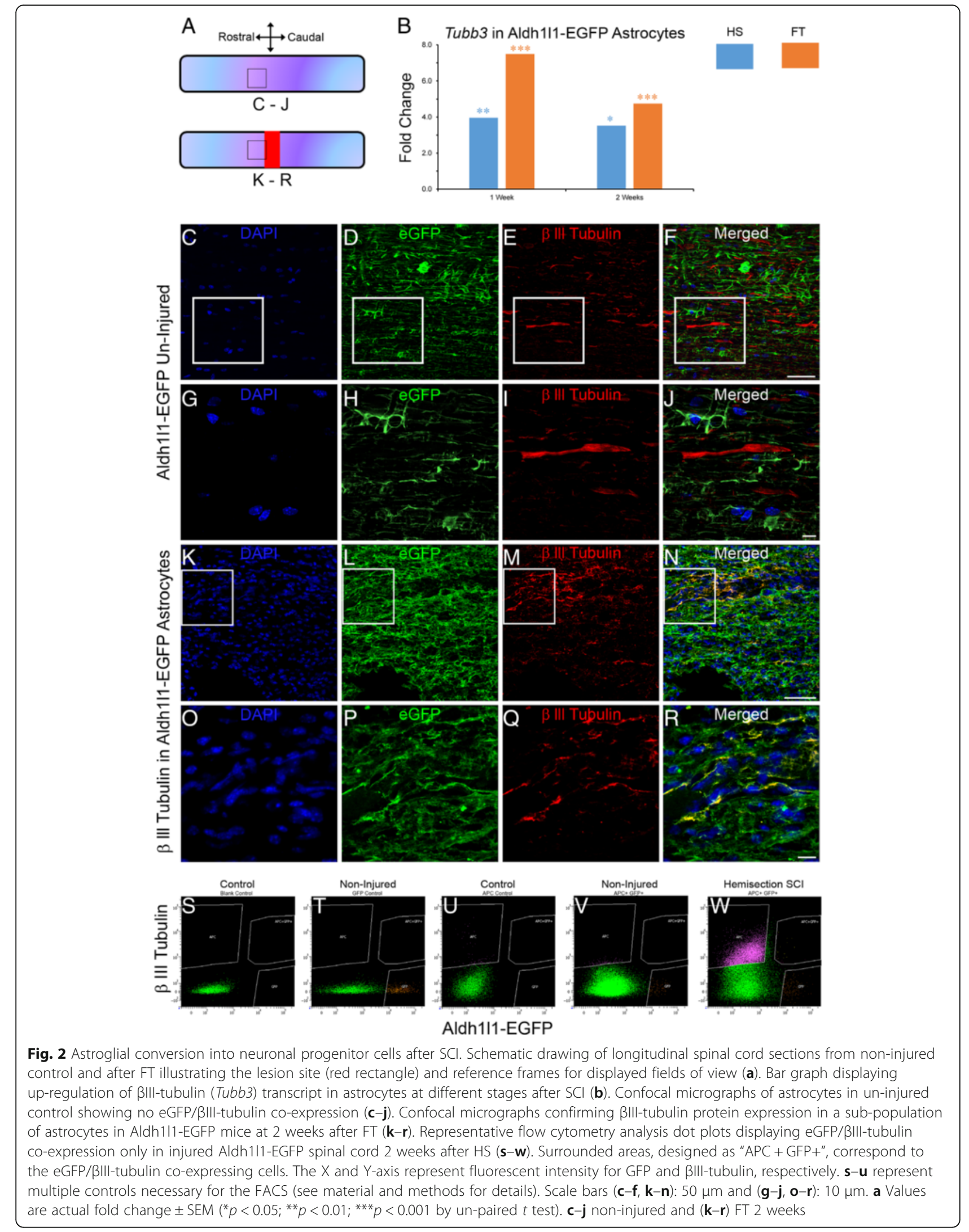


and found no BrdU incorporation in eGFP/BIII-tubulin co-expressing cells, suggesting that these cells are derived from resident mature astrocytes (Fig. $3 \mathrm{a}-\mathrm{g}$ ). To determine whether transdifferentiated astrocytes express other neuronal progenitor markers, we used doublecortin immunostaining (DCX, another classical neuronal progenitor marker) and found that eGFP/ $\beta$ III-tubulin co-expressing cells also express DCX (Fig. $4 \mathrm{f}-\mathrm{j}$ ). Astrocytes in non-injured controls showed no BIII-tubulin or DCX expression (Fig. 4a-e).

To examine whether astrocytes-derived neuronal progenitors are subsequently converted into neurons, we

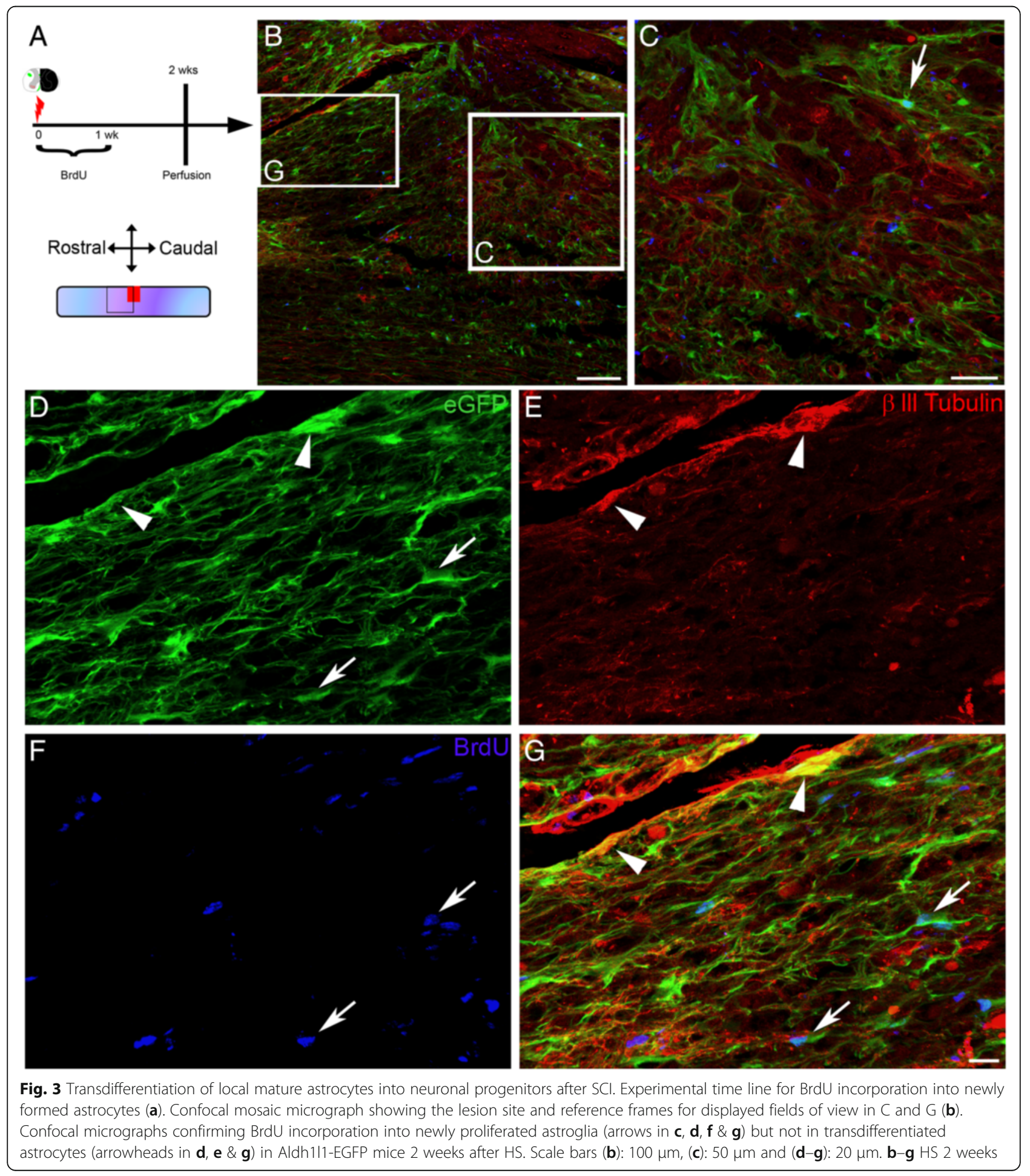




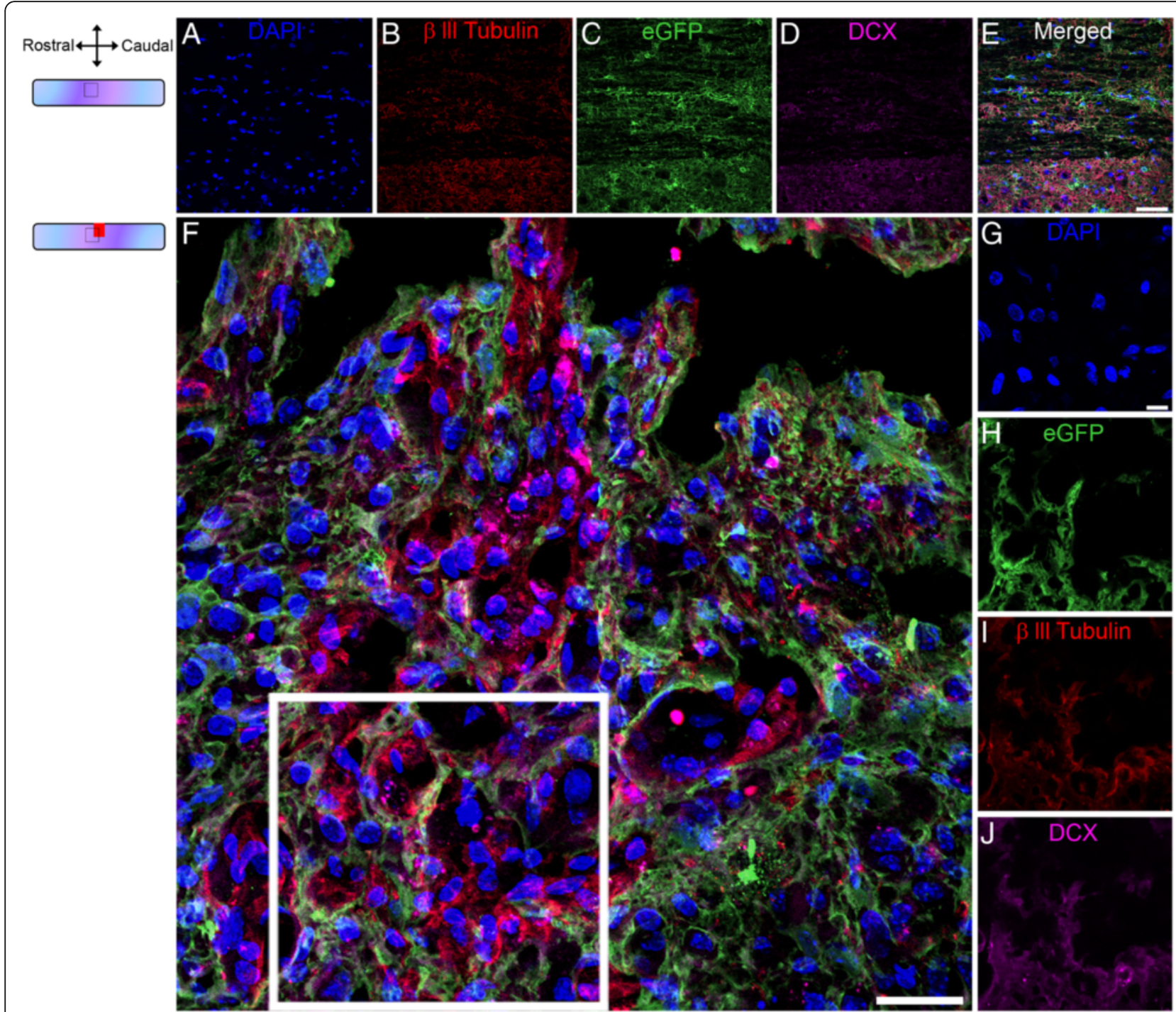

Fig. 4 Doublecortin expression in astrocytes after SCI. Confocal micrographs showing DAPI (a), Blll-tubulin (b), astrocytes (eGFP, c) and DCX (d) expression in un-injured mice. No ßlll-tubulin/eGFP/DCX co-expression was observed in un-injured astrocytes (e). Following SCI, transdifferentiated (eGFP/BIII-tubulin positive) astrocytes also expressed DCX (f $\& \mathbf{h}-\mathbf{j})$. DAPI (g). Scale bars $(\mathbf{a}-\mathbf{e}): 50 \mu \mathrm{m},(\mathbf{f}): 25 \mu \mathrm{m},(\mathbf{g}-\mathbf{j}): 10 \mu \mathrm{m}$. $\mathbf{a}-\mathbf{e}$ non-injured and $(\mathbf{f}-\mathbf{j})$ HS 2 weeks

performed immunohistochemistry to detect parvalbumin and glutamate decarboxylase (GAD 65/67) as markers for mature inhibitory GABAergic interneurons, as well as T-cell leukemia homeo box 3 (Tlx3/Rnx) and neuronal nucleus (NeuN) as glutamatergic and mature neuronal markers in Aldh1l1-EGFP mice (Figs. 5 and 6). In non-injured animals, astrocytes displayed typical stellate morphology with no parvalbumin or GAD 65/67 expression (Fig. 5a-d). Following lesion astrocytes displayed elongated morphology and showed parvalbumin expression within somata and primary processes (Fig. 5e-k). High magnification confocal analysis confirmed eGFP/parvalbumin co-expression in Aldh1L1EGFP mice after lesion (Fig. 5j, k). The GABAergic interneuron identity of astrocytes-derived cells was further demonstrated by staining with antibody against GAD 65/67 (Fig. 5h, i). SCI-induced reactive astrocytes did not express glutamatergic or mature neuronal markers such as Tlx3/Rnx (Fig. 6a-h) and NeuN (Fig. 6i-r).

To investigate the potential mechanisms responsible for astroglial transdifferentiation, we analyzed the expression of neurogenic markers in astrocytes after injury. We observed an increased expression of the gene encoding for fibroblast growth factor receptor 4 (Fgfr 4 ), a neural stem cell marker [17], at 1 and 2 weeks after both lesion severities (Fig. 7a). Morphologically, Fgfr4 protein expression was evident in elongated astrocytic 


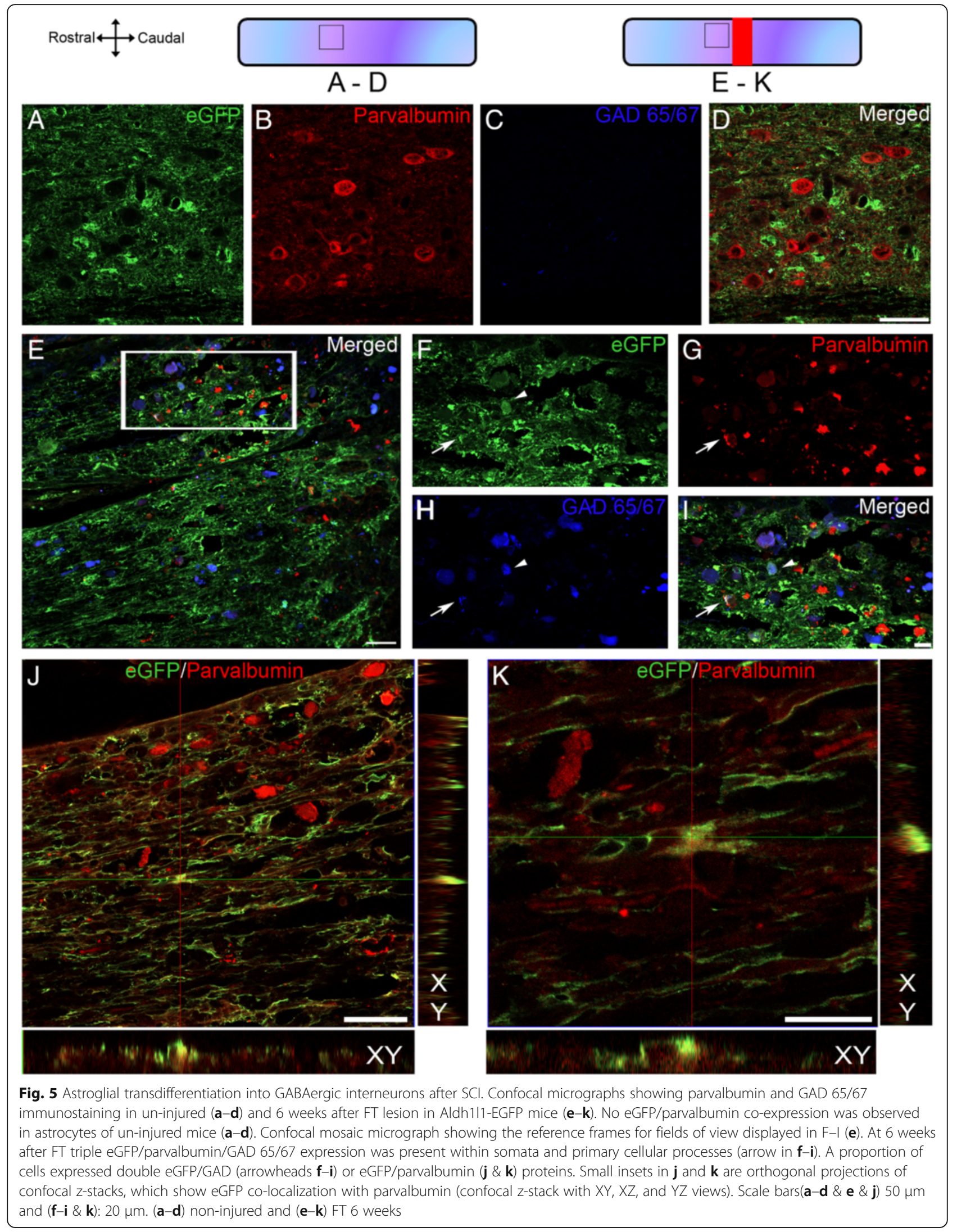




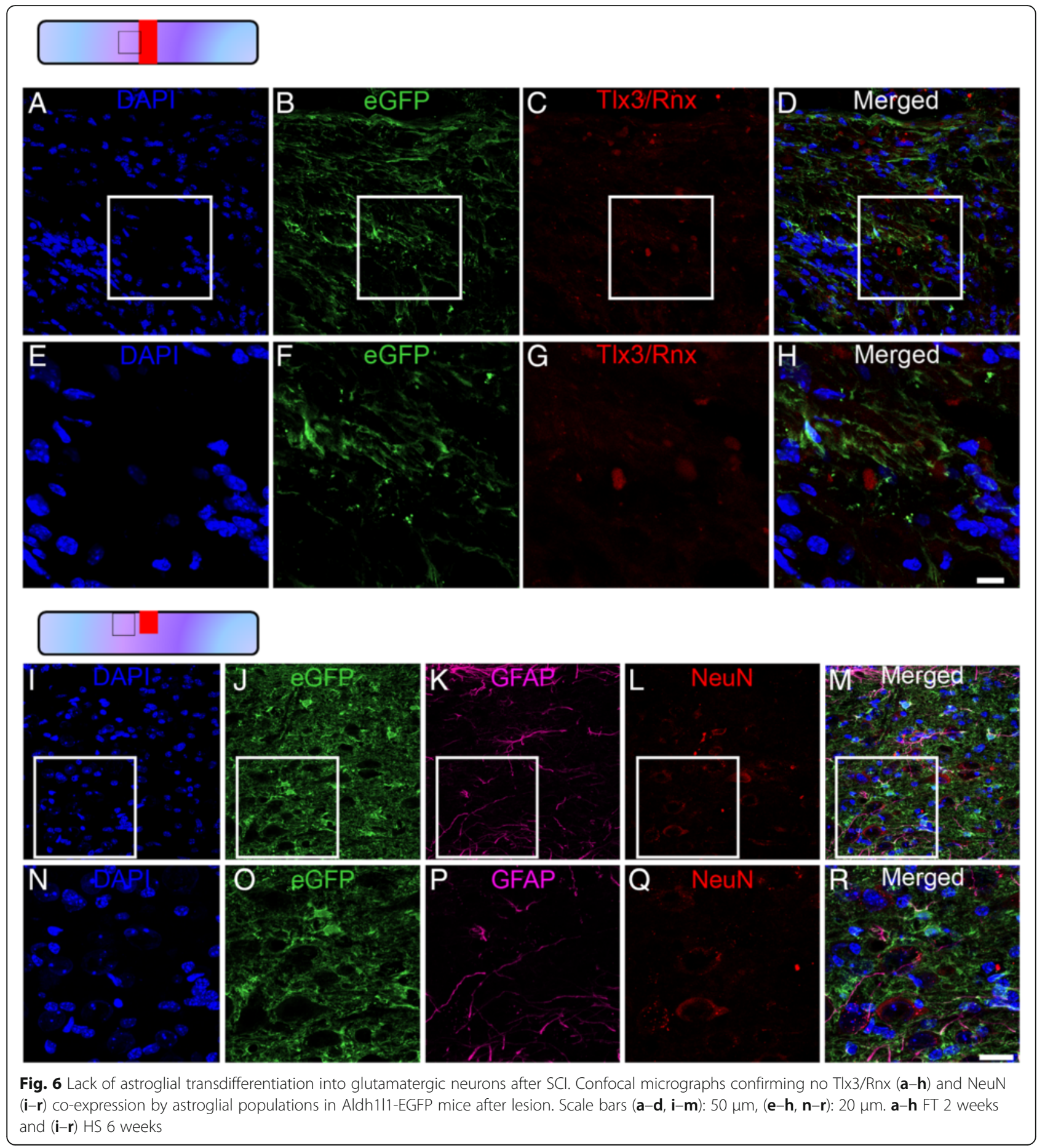

cytoskeleton and closely matched GFAP expressing profiles in non-injured control (Fig. 7b-k) and injured spinal cord (Fig. 7l-u). SCI triggered a clear increase in Fgfr4 expression in astrocytes located adjacent to the lesion site (Fig. 7o). Average Fgfr4 area fraction expression $1000 \mu \mathrm{m}^{2}$ rostral and $1000 \mu^{2}$ caudal to the lesion site revealed significant increase compared to non-injured throughout 6 weeks after FT injury (Fig. 7v). The peak in Fgfr4 protein expression was observed at $72 \mathrm{~h}$ with over 8 -fold increase in area fraction compared to non-injured control (Fig. 7v). Further analysis revealed greater increase in Fgfr4 expression rostral compared to caudal segment of the lesion site (Fig. 7w, x). 
A

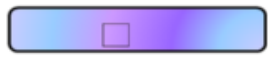

B - F

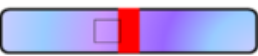

$\mathrm{L}-\mathrm{U}$

Fgfr4 in Aldh111-EGFP Astrocytes

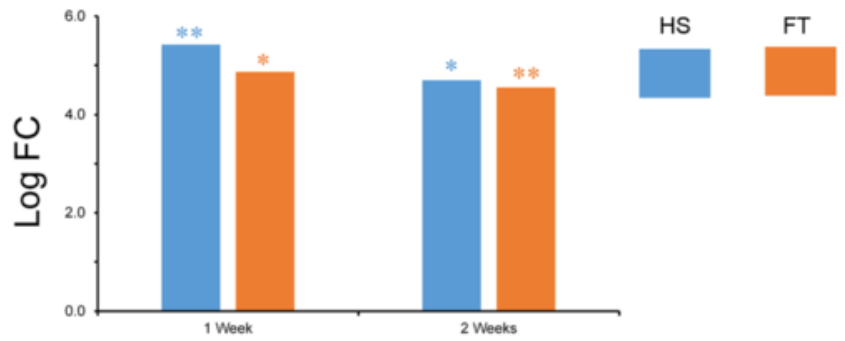

B
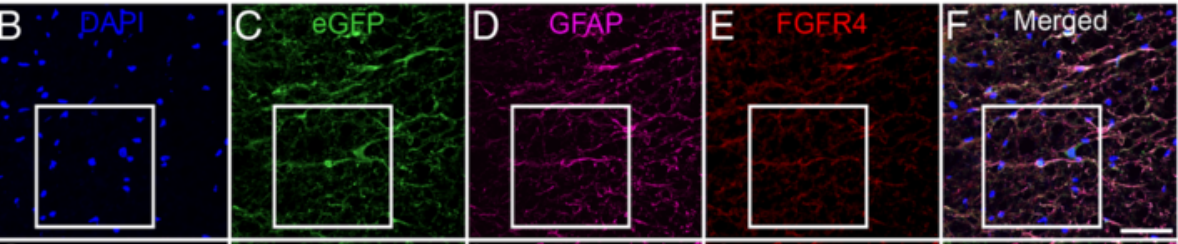

G
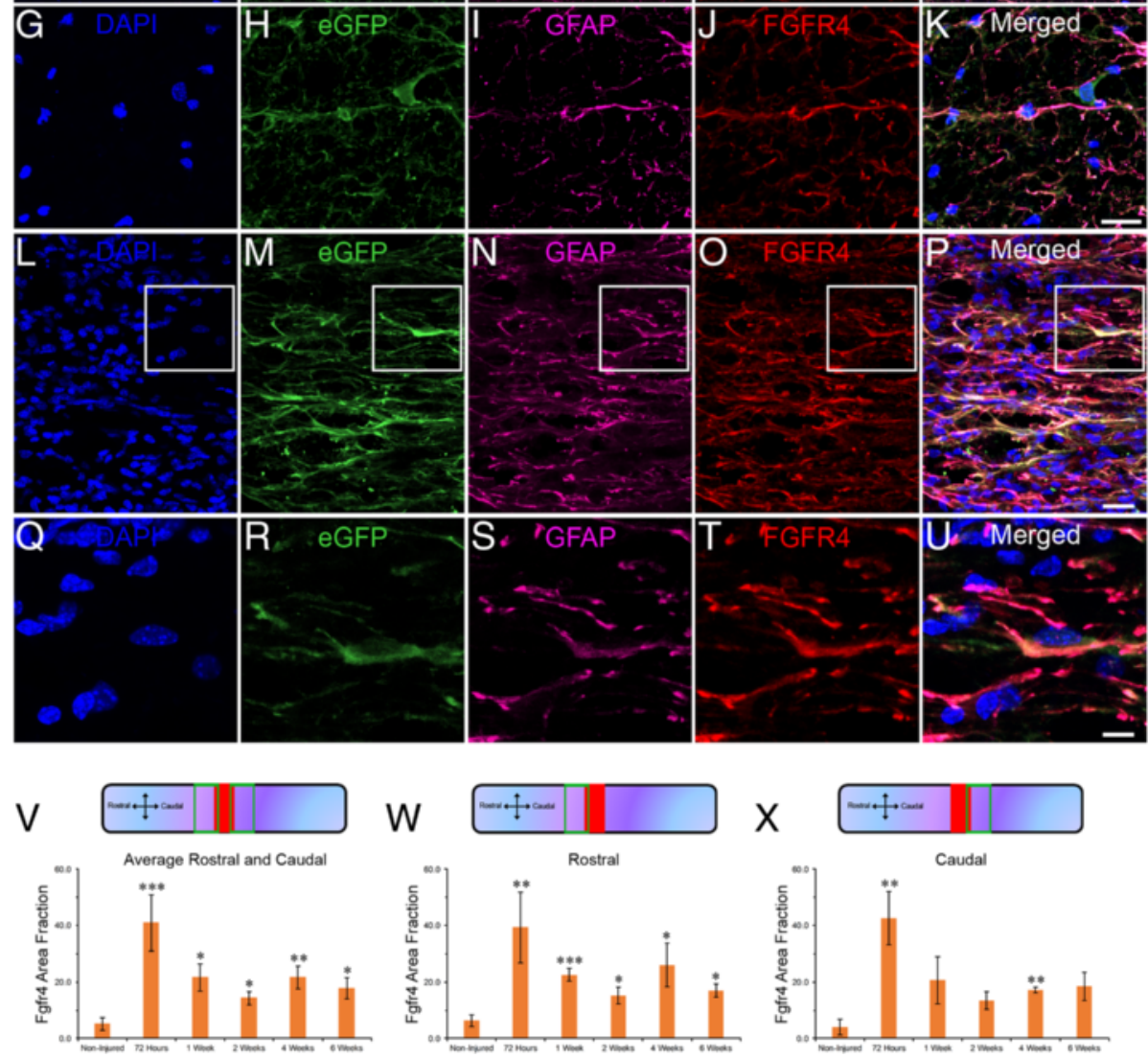

Fig. 7 Up-regulation of Fgfr4 in astrocytes after SCl. Bar graph displaying increased Fgfr4 transcript expression in astrocytes (a). Confocal micrographs confirming astrocytic Fgfr4 protein over-expression after injury in Aldh1l1-EGFP mice (I-u) as compared to un-injured mice (b-k). Morphologically, Fgfr4 expression was identical to GFAP and eGFP profiles in Aldh1/1-EGFP mice. DAPI staining (b, g, I \& $\mathbf{q}$ ). Bar graphs displaying increased Fgfr4 protein expression at multiple stages after FT injury (v-x). Note that injury-induced increase in Fgfr4 protein expression is shown

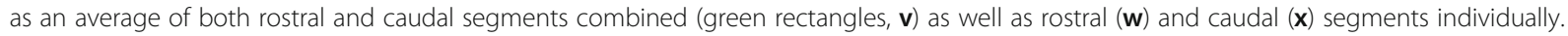
Scale bars (b-f): $50 \mu \mathrm{m},(\mathbf{g}-\mathbf{k}): 20 \mu \mathrm{m},(\mathbf{I}-\mathbf{p}): 25 \mu \mathrm{m},(\mathbf{q}-\mathbf{u}): 10 \mu \mathrm{m}$. a Values are actual fold change \pm SEM $\left({ }^{*} p<0.05 ;{ }^{* *} p<0.01\right.$ by $t$ test). $\mathbf{v}-\mathbf{x}$ Values are area fractions \pm SEM $\left({ }^{*} p<0.05 ;{ }^{* *} p<0.01 ;{ }^{* *} p<0.001\right.$ by un-paired $t$ test). b-k un-injured and (I-u) FT 4 weeks 
Altogether, these data demonstrate SCI-induced astrocytic conversion towards neuronal progenitor and subsequently GABAergic interneurons lineage. Astrocytic transdifferentiation is strongly associated with over-expression of the neural stem cell marker Fgfr4.

\section{Glial transdifferentiation continues up to 6 weeks post-lesion}

We finally examined SCI-induced astroglial transdifferentiation in adult spinal cord at multiple stages after different lesion severity (Fig. 8). Time-course analyses in Aldh1l1-EGFP mice revealed that astrocytic co-expression of the BIII-tubulin protein starts as early as $72 \mathrm{~h}$ following injury and showed a peak between 1 and 2 weeks post-lesion reaching over $11.1 \%$ after FT injury (Fig. 8f). By 6 weeks after lesion only 2.9 and $1.2 \%$ of astrocytes continued to express $\beta$ III-tubulin in HS and FT groups, respectively (Fig. 8f).

These data demonstrate that astrocyte transdifferentiation towards neuronal lineage starts early after injury peaking by 1 week post-lesion and continues to a lower level up to 6 weeks (the longest time-point we have investigated in this study) following both injury severities.

\section{Discussion}

Findings of the current study indicate that astrocytes undergo specific transcriptomic alterations after SCI that depends on both time post-lesion and injury severity. Importantly, SCI induces astrocytic conversion (both morphologically and via $\beta$ III-tubulin and DCX expressions) towards neuronal progenitors and subsequently GABAergic interneurons (see Fig. 9). Increased astroglial transdifferentiation is associated with over-expression of the neural stem cell marker Fgfr4 and continues up to 6 weeks after CNS injury. These findings represent the first cell-specific transcriptomic analysis of astrocytes after different lesion severities providing a novel insight into astroglial plasticity.

\section{Heterogeneous astrocytic response after $\mathrm{SCl}$}

Astrogliosis is a pathological hallmark of SCI involving not only morphological hypertrophy but also pronounced changes at molecular and functional levels $[2,18]$. Contradictory reports on astrocytic response after SCI has been reported including increased [3] and inhibition of neuroinflammation [4]. Our data provide the first evidence of a heterogeneous astrocytic response
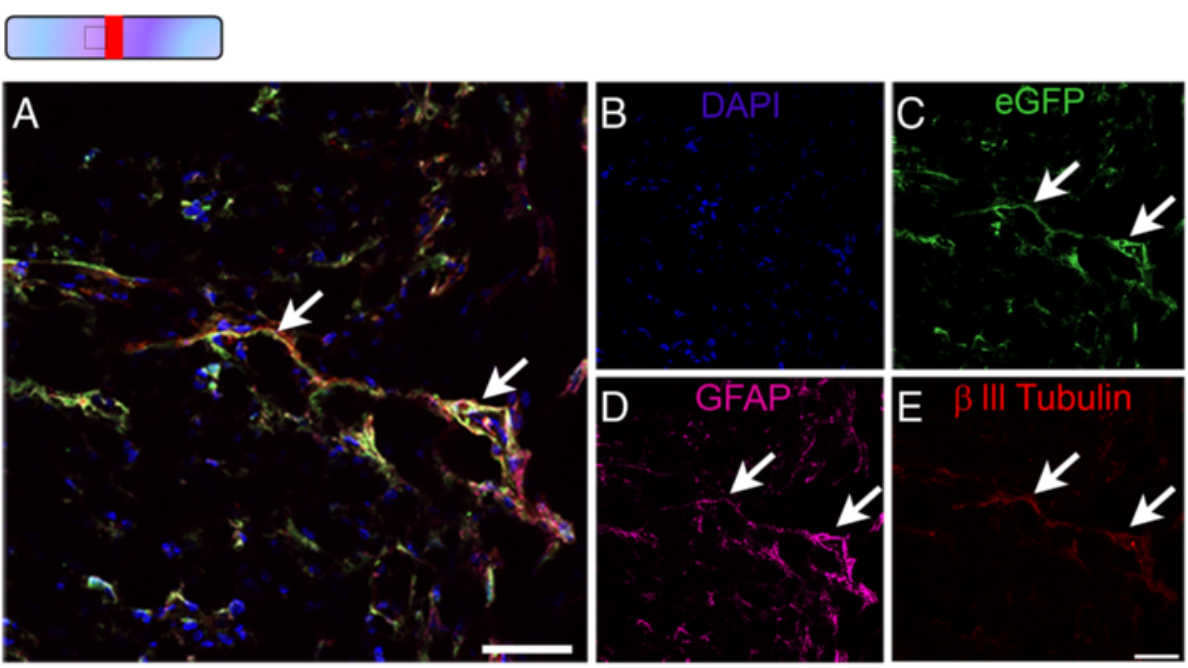

$\mathrm{F}$

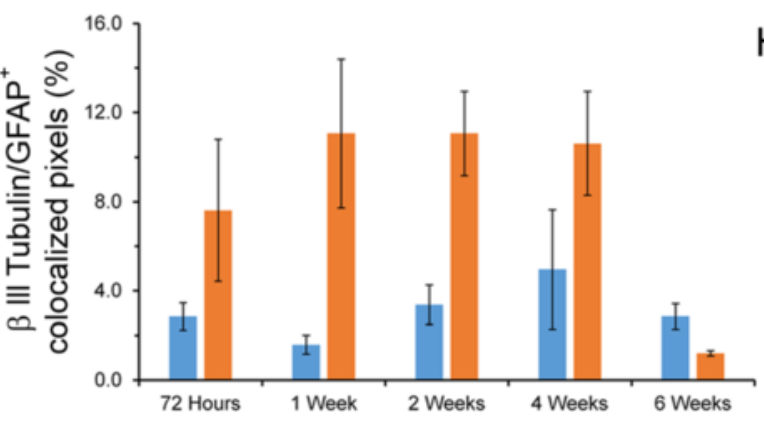

Fig. 8 Astroglial transdifferentiation continues up to 6 weeks after different lesion severity. Confocal micrographs showing merged of DAPI (b), eGFP (c), GFAP (d) and BIII-tubulin (e) expression in Aldh1I1-EGFP mice 4 weeks after FT injury (a). Quantitative analysis of GFAP/BIII-tubulin (f) co-expressing cells (arrows) in the 2 lesion severities at multiple stages after SCl. Scale bars (a-e): $50 \mu \mathrm{m}$. Bars represent mean \pm SEM. a-e FT 4 weeks 


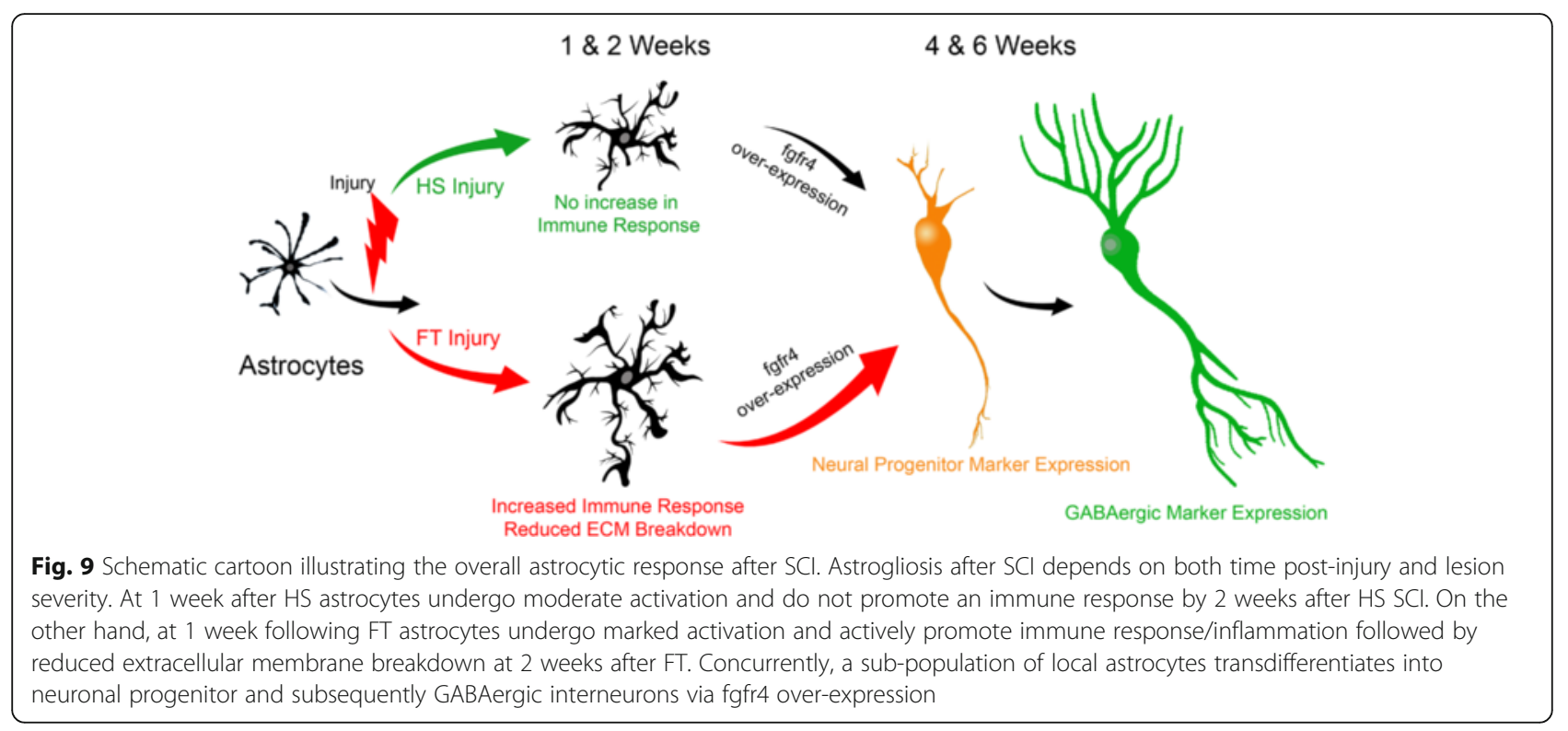

after SCI that depends on both time post-lesion and injury severity. Specifically, astrogliosis after FT may be detrimental for regeneration by promoting neuroinflammation (1 week) and inhibiting extracellular remodeling ( 2 weeks), whereas astrocytic response after HS may be beneficial by an absence of increase (1 week) and down-regulation of immune response ( 2 weeks). Our data provide the first evidence for the general consensus that astrocytic response after SCI depends on numerous factors including lesion severity, time post-injury as well as distance to the lesion epicenter [2, 18]. Heterogeneous astrocytic response has been reported after lipopolysaccharide injection and stroke [14] as well as at different stages of Alzheimer's disease [13]. Altogether, these findings demonstrate that astrogliosis depends on multiple factors including the type of inducing stimulus, time after activation and injury severity.

\section{Transdifferentiation of astrocytes towards neuronal lineage after $\mathrm{SCl}$}

Another important finding from our study is the autologous transdifferentiation of resident mature astrocytes into neuronal progenitors and subsequently GABAergic interneurons after SCI. Previous studies have reported SCI-induced expression of progenitor markers by astrocytes including Sox2 and nestin [19, 20]. However, contrary to our findings, no study had shown spontaneous astroglial conversion into neuronal progenitors or adult neurons. One reason for this discrepancy may be due to markers used to label astrocytes. All the mentioned studies had relied on GFAP as a marker of astrocytes, whereas we have used Aldh1L1 expression that has been shown to be a more pan astrocytic marker than
GFAP [15]. Our findings provide evidence that a small population of resident astrocytes are spontaneously reprogrammed towards GABAergic interneurons in vivo after CNS injury. Lack of BrdU incorporation into eGFP//IIItubulin co-expressing cells confirmed that the origin of these cells are transdifferentiated resident astrocytes rather than newly formed astrocytes. Comparison between the two lesion severity showed greater level of eGFP//IIItubulin co-expression after severe SCI, suggesting that greater astrocytic activation and enlarged lesion area may also play important roles in astrocyte transdifferentiation toward neuronal lineage.

More recent findings suggest that enforced expression of neurogenic factors including Notch [21], Sox2 [22], NeuroD1 [23], Shh [24], Ascl1, Brn2 and Myt1l [25] can directly convert astrocytes into functional neurons in vivo. However, we found no significant over expression of these transcripts, suggesting that other signaling molecules may be involved in autologous astrocytic transdifferentiation towards neuronal lineage after SCI. In this regard, we have identified the fibroblast growth factor receptor $4(F g f r 4)$ as a potential autologous modulator of astrocytic transdifferentiation following SCI. This is, to our knowledge, the first demonstration that Fgfr4 protein is expressed in astroglial cells and its expression is increased in activated astrocytes following CNS injury. Interestingly, the peak increase in Fgfr4 protein expression in astrocytes was observed at $72 \mathrm{~h}$ post-lesion, whilst the peak increase in BIII-tubulin protein expression was not observed till 1 week post-lesion, suggesting that Fgfr4 protein over-expression precedes astroglial conversion towards neuronal lineage.

The 4 subfamilies of fibroblast growth factor receptors (Fgfr1-4) play multiple functions in progenitor cell 
proliferation and neuronal differentiation [26]. Endogenously expressed Fgfr4 ligand (Fgf4) promotes embryonic stem cell differentiation towards neuronal lineage [27] and elevated Fgf4 is essential for astrocytic conversion into neuronal progenitor-like cells [28]. We found no changes in Fgfr 1 and Fgfr2 transcripts, whilst the gene encoding for Fgfr3 was actually down-regulated 1 week after HS injury. Taken together, these findings suggest that Fgf4 action on astrocytes is most likely due to the activation of Fgfr4 rather than other fgf receptor family members. Elevated Fgfr4 expression in astrocytes may thus be critical for their transdifferentiation into neuronal progenitor-like cells after SCI.

A major difference between our findings and published reports is that whereas others had introduced extrinsic neurogenic factors to force astrocyte transformation into neurons, we observed here a spontaneous phenomenon. Equally important, none of the previous studies had shown time-course analysis of glial transdifferentiation in vivo. Altogether, these findings suggest that optimizing autologous glial transdifferentiation may be more effective in replacing neuronal loss and improving functional recovery following injury. The functional effect of astroglial transformation into neurons awaits further studies.

\section{Conclusion}

Our data highlight that astrocytic response after injury is both time -and severity-dependent involving inhibition and stimulation of immune response after moderate and severe SCI, respectively. Importantly, we show that SCI induces astrocytic conversion towards GABAergic interneurons via transition through neural stem cell (Fgfr4) and neuronal progenitors ( $\beta$ III-tubulin/DCX) stages. Further studies are required to establish whether astrocytederived interneurons can form functional synapses with the neighboring neurons and facilitate neurotransmission across the lesion site. Enforced Fgfr4 and BIII-tubulin expression in reactive astrocytes may promote greater astroglial transdifferentiation towards neuronal lineage and provide a novel therapeutic approach to replace neuronal demise and promote functional recovery after CNS injury.

\section{Additional files}

Additional file 1: Figure S1. Specific eGFP expression of astroglial cells in Aldh111-EGFP mice. Confocal micrographs showing astrocytic eGFP expression in Aldh111-EGFP mice that was confirmed using GFAP immunostaining $(A-C)$. Fluorescent micrographs showing lack of eGFP expression in microglia/macrophages (Iba1, D\&E), oligodendrocytes (MBP, F) and neuronal (MAP2, G) populations in Aldh111-EGFP mice. Scale bars (A, F\&G): $50 \mu \mathrm{m}$, (B\&C): $10 \mu \mathrm{m},(\mathrm{D}): 30 \mu \mathrm{m},(\mathrm{E}): 15 \mu \mathrm{m}$. Representative flow cytometry analysis dot plot displaying control $(\mathrm{H})$ and eGFP-expressing astrocytic profiles from un-injured (I) as well as after injury (J). Surrounded areas, designed as "P4", correspond to the eGFP-expressing astrocytes. The $X$-axis represents fluorescent intensity and the $Y$ axis cell size. RNA quality isolated from FACSed astrocytes (K). (TIF $20779 \mathrm{~kb}$ )
Additional file 2: Table S1. Database of differential expression comparison of activated astrocyte RNA-Seq data relative to non-injured control astrocyte at 1 and 2 weeks after hemisection and full transection injuries. (XLSX $90 \mathrm{~kb}$ )

Additional file 3: Table S2. Pathway analysis of differentially expressed genes in activated astrocytes after hemisection and full transection injuries. (XLSX $19 \mathrm{~kb}$ )

Additional file 4: Figure S2. Induction of transdifferentiation pathways in astrocytes after SCl. Gene ontology pathway map analysis of deregulated genes in astrocytes demonstrate the induction of a neural development pathway. Thermometers indicate the deregulation of the gene (red: up-regulated; blue: down-regulated). Thermometers with "1" represent gene deregulation in astrocytes. Interactions between objects: green (positive or activation); red (negative or inhibition); grey (unspecified): B: Binding (physical interaction between molecules). (TIF $3235 \mathrm{~kb}$ )

\section{Abbreviations}

7-AAD: 7-aminoactinomycin; Aldh1L1: Aldehyde dehydrogenase 1 family member L1; DCX: Doublecortin; eGFP: Enhanced green fluorescent protein; FACS: Fluorescence-activated cell sorting; Fgfr4: Fibroblast growth factor receptor 4; FT: Full transection; GAD 65/67: Glutamate decarboxylase; HS: Hemisection; MMRRC: Mutant Mouse Regional Resource Centre; NeuN: Neuronal nucleus; NI: Non-injured; PBS: Phosphate base saline; PFA: Paraformaldehyde; RNA-Seq: RNA sequencing; SCl: Spinal cord injury; Tlx3/Rnx: T-cell leukemia homeo box 3

\section{Acknowledgements}

We thank Dr. N. Heintz, The Rockefeller University, USA that donated the Aldh111-EGFP mice to the MMRRC. We thank M. Rabano and M. dM Vivanco for technical assistance in FACS samples collection. Transcriptomic experiments were done at iGE3Genomics Platform, University of Geneva Switzerland; we thank in particular M. Docquier and C. Delucinge for their assistance in transcriptomic analysis. Histological analysis was done at Montpellier Rio Imaging platform. Finally, we thank A. Webb for correction of the English text.

\section{Fundings}

This work was supported by the Spanish Government, Plan Nacional de I + D + I 2008-2011 and ISCIII- Subdirección General de Evaluación y Fomento de la investigación (PI10/00709) [to FEP] and the Government of the Basque Country grant (Proyectos de Investigacion Sanitaria and Fondo Comun de Cooperacion Aquitania-Euskadi) [to FEP], the French Government, ANR-FNS grant, GliALS (№ ANR-14-CE36-0009-01) [to FEP], the patient organizations "Demain Debout Aquitaine" [to YNG and HNN] and "Verticale" [to FEP and HNN]. The authors declare no conflicts of interests.

\section{Availability of supporting data}

All data analysed during this study are included in this published article and its supplementary information files; raw genomic datasets available from the corresponding author on reasonable request.

\section{Authors' contributions}

HNN participated in the design of the study, performed all experiments, analyzed the data and contributed to the writing of the manuscript; JCS participated in $\mathrm{SCl}$ and FACS; HB participated in image acquisition, ECS participated in SCl; YNG participated in SCl and data analysis, and FEP conceptualized the research, designed the project, participated in the analysis and interpretation of data, drafting the work and final approval. All authors read and approved the final manuscript.

\section{Authors' information}

Not applicable.

\section{Competing interests}

The authors declare that they have no competing interests. 


\section{Ethics approval and consent to participate}

Experimental procedures followed the European legislative, administrative and statutory measures for animal experimentation (86/609/EEC). The study was approved by the "Direction des Services Vétérinaires de l'Hérault" and the "Ministère de l'éducation nationale, de l'enseignement supérieur et de la recherche, ethic committee N³6", France (authorization number 34118).

\section{Author details}

'University of Montpellier, Montpellier F-34095, France. ${ }^{2}$ INSERM U1198, Place Eugène Bataillon CC105, 34095, Montpellier Cedex 5, France. ${ }^{3}$ EPHE, Paris F-75014, France. ${ }^{4}$ INSERM U1051, F-34095 Montpellier, France. ${ }^{5}$ Integrative Biology of Neurodegeneration", IKERBASQUE Basque Foundation for Science and Neuroscience Department, University of the Basque Country, E-48013 Bilbao, Spain. ${ }^{6}$ Department of Neurosurgery, Gui de Chauliac Hospital, F-34295 Montpellier, France.

\section{Received: 11 March 2016 Accepted: 28 September 2016} Published online: 06 October 2016

\section{References}

1. Fawcett JW, Asher RA. The glial scar and central nervous system repair. Brain Res Bull. 1999:49:377-91.

2. Karimi-Abdolrezaee S, Billakanti R. Reactive astrogliosis after spinal cord injury-beneficial and detrimental effects. Mol Neurobiol. 2012;46:251-64.

3. Brambilla R, Hurtado A, Persaud T, Esham K, Pearse DD, Oudega M, Bethea JR. Transgenic inhibition of astroglial NF-kappa B leads to increased axonal sparing and sprouting following spinal cord injury. J Neurochem. 2009;110:765-78.

4. Kim JH, Min KJ, Seol W, Jou I, Joe EH. Astrocytes in injury states rapidly produce anti-inflammatory factors and attenuate microglial inflammatory responses. J Neurochem. 2010:115:1161-71.

5. Wanner IB, Anderson MA, Song B, Levine J, Fernandez A, Gray-Thompson Z, Ao Y, Sofroniew MV. Glial scar borders are formed by newly proliferated, elongated astrocytes that interact to corral inflammatory and fibrotic cells via STAT3dependent mechanisms after spinal cord injury. J Neurosci. 2013;33:12870-86.

6. Beutner C, Linnartz-Gerlach B, Schmidt SV, Beyer M, Mallmann MR, Staratschek-Jox A, Schultze JL, Neumann H. Unique transcriptome signature of mouse microglia. Glia. 2013;61:1429-42.

7. Chiu IM, Morimoto ET, Goodarzi H, Liao JT, O'Keeffe S, Phatnani HP Muratet M, Carroll MC, Levy S, Tavazoie S, et al. A neurodegeneration-specific gene-expression signature of acutely isolated microglia from an amyotrophic lateral sclerosis mouse model. Cell Rep. 2013;4:385-401.

8. Hickman SE, Kingery ND, Ohsumi TK, Borowsky ML, Wang LC, Means TK, El Khoury J. The microglial sensome revealed by direct RNA sequencing. Nat Neurosci. 2013;16:1896-905.

9. Huang C, Huang B, Bi F, Yan LH, Tong J, Huang J, Xia XG, Zhou H. Profiling the genes affected by pathogenic TDP-43 in astrocytes. J Neurochem. 2014;129:932-9.

10. Noristani HN, Sabourin JC, Gerber YN, Teigell M, Sommacal A, Vivanco M, Weber M, Perrin FE. Brca1 is expressed in human microglia and is dysregulated in human and animal model of ALS. Mol Neurodegener. 2015;10:34.

11. Olah M, Amor S, Brouwer N, Vinet J, Eggen B, Biber K, Boddeke HW. Identification of a microglia phenotype supportive of remyelination. Glia. 2012;60:306-21.

12. Parakalan $R$, Jiang B, Nimmi B, Janani M, Jayapal M, Lu J, Tay SS, Ling EA, Dheen ST. Transcriptome analysis of amoeboid and ramified microglia isolated from the corpus callosum of rat brain. BMC Neurosci. 2012;13:64

13. Simpson JE, Ince PG, Shaw PJ, Heath PR, Raman R, Garwood CJ, Gelsthorpe C, Baxter L, Forster G, Matthews FE, et al. Microarray analysis of the astrocyte transcriptome in the aging brain: relationship to Alzheimer's pathology and APOE genotype. Neurobiol Aging. 2011;32:1795-807.

14. Zamanian JL, Xu L, Foo LC, Nouri N, Zhou L, Giffard RG, Barres BA. Genomic analysis of reactive astrogliosis. J Neurosci. 2012;32:6391-410.

15. Cahoy JD, Emery B, Kaushal A, Foo LC, Zamanian JL, Christopherson KS, Xing Y, Lubischer JL, Krieg PA, Krupenko SA, et al. A transcriptome database for astrocytes, neurons, and oligodendrocytes: a new resource for understanding brain development and function. J Neurosci. 2008;28:264-78.

16. Noristani HN, Lonjon N, Cardoso M, Le Corre M, Chan-Seng E, Captier G, Privat A, Coillot C, Goze-Bac C, Perrin FE. Correlation of in vivo and ex vivo (1)H-MRI with histology in two severities of mouse spinal cord injury. Front Neuroanat. 2015;9:24.
17. Limke TL, Cai J, Miura T, Rao MS, Mattson MP. Distinguishing features of progenitor cells in the late embryonic and adult hippocampus. Dev Neurosci. 2003;25:257-72.

18. Sofroniew MV. Molecular dissection of reactive astrogliosis and glial scar formation. Trends Neurosci. 2009;32:638-47.

19. Wanner IB, Mahoney J, Jessen KR, Wood PM, Bates M, Bunge MB. Invariant mantling of growth cones by Schwann cell precursors characterize growing peripheral nerve fronts. Glia. 2006;54:424-38.

20. White RE, McTigue DM, Jakeman LB. Regional heterogeneity in astrocyte responses following contusive spinal cord injury in mice. J Comp Neurol. 2010:518:1370-90.

21. Magnusson JP, Goritz C, Tatarishvili J, Dias DO, Smith EM, Lindvall O, Kokaia Z, Frisen J. A latent neurogenic program in astrocytes regulated by Notch signaling in the mouse. Science. 2014;346:237-41.

22. Su Z, Niu W, Liu ML, Zou Y, Zhang CL. In vivo conversion of astrocytes to neurons in the injured adult spinal cord. Nat Commun. 2014;5:3338

23. Guo Z, Zhang L, Wu Z, Chen Y, Wang F, Chen G. In vivo direct reprogramming of reactive glial cells into functional neurons after brain injury and in an Alzheimer's disease model. Cell Stem Cell. 2014:14:188-202.

24. Sirko S, Behrendt G, Johansson PA, Tripathi P, Costa M, Bek S, Heinrich C, Tiedt S, Colak D, Dichgans $M$, et al. Reactive glia in the injured brain acquire stem cell properties in response to sonic hedgehog. Cell Stem Cell. 2013;12:426-39.

25. Torper O, Pfisterer U, Wolf DA, Pereira M, Lau S, Jakobsson J, Bjorklund A Grealish S, Parmar M. Generation of induced neurons via direct conversion in vivo. Proc Natl Acad Sci U S A. 2013:110:7038-43.

26. Guillemot F, Zimmer C. From cradle to grave: the multiple roles of fibroblast growth factors in neural development. Neuron. 2011;71:574-88.

27. Kunath T, Saba-El-Leil MK, Almousailleakh M, Wray J, Meloche S, Smith A. FGF stimulation of the Erk1/2 signalling cascade triggers transition of pluripotent embryonic stem cells from self-renewal to lineage commitment. Development. 2007;134:2895-902.

28. Feng GD, He BR, Lu F, Liu LH, Zhang L, Chen B, He ZP, Hao DJ, Yang H. Fibroblast growth factor 4 is required but not sufficient for the astrocyte dedifferentiation. Mol Neurobiol. 2014;50:997-1012.

\section{Submit your next manuscript to BioMed Central and we will help you at every step:}

- We accept pre-submission inquiries

- Our selector tool helps you to find the most relevant journal

- We provide round the clock customer support

- Convenient online submission

- Thorough peer review

- Inclusion in PubMed and all major indexing services

- Maximum visibility for your research

Submit your manuscript at www.biomedcentral.com/submit
C) Biomed Central 